\title{
New challenges to mental health needs in response to the COVID-19 outbreak in India: Insights from a pilot survey
}

Suman Ray ( $\nabla$ sumanitrc@gmail.com )

CSIR-National Institute of Science Technology and Development Studies (CSIRNISTADS)

\section{Short Report}

Keywords: COVID-19 outbreak, mental health, anxiety and stress

Posted Date: October 5th, 2020

DOI: https://doi.org/10.21203/rs.3.rs-86147/v1

License: (c) (1) This work is licensed under a Creative Commons Attribution 4.0 International License.

Read Full License 


\section{Abstract}

With the pandemic rapidly rising at the global level and so its impact on the mental health, a pilot survey (online) using DASS Scale 21 was conducted to know how COVID-19 has affected mental health status of general population in India. The results showed that anxiety and stress were prevalent from mild to moderate level and depression in mild level. As the uncertain future due to COVID-19 is becoming dominant, it is high time to prepare ourselves so as to prevent the second wave of mental health crises post COVID-19 pandemic.

\section{Background}

COVID-19 outbreak was first declared as pandemic by WHO on March 11, 2020. The evolution of COVID19 being unpredictable and vaccine trial process still ongoing and no cure to this has certainly increased the anxiety among the general population. With social distancing is the key to minimise the disease spread, associated lockdown strategy with schools being in online mode and downturn of the economy and other factors such as restricted movements with no outdoor activities altogether etc. has exacerbated the risk of mental health problems. COVID-19 pandemic has led to various concerns regarding its mental health effect on patients with psychiatric disorders and also the health-care workforce too. Psychological distress among those already suffering from psychiatric syndromes is on rise due to coronavirus-induced lockdown, isolation and quarantine recently reported in India (Aguiar Melo and de Sousa Soares, 2020). As per WHO, India has a population of 1,324,171,00 and total expenditure on health as \% of GDP (2014) is 4.7. Mental health workforce in India (per 100,000 population) include psychiatrists $(0.3)$, nurses $(0.12)$, psychologists (0.07) and social workers (0.07). WHO estimates that the burden of mental health problems in India is of the tune of 2,443 DALYs per 100,000 population, and the economic loss, due to mental health conditions, between 20122030, is 1.03 trillions of 2010 dollars. Treatment gap for mental disorders still remains very high in India as per National Mental Health Survey of India, 2015-2016 report. Status of mental disorders in India is alarming as National Mental Health Survey of India, report 20152016. Mental disorders has been indicated as one of the leading causes of non-fatal disease burden in India (Dandona, 2020). The needs of vulnerable groups, including those with severe mental illness, learning difficulties, and neuro developmental disorders etc. due to COVID-19 pandemic need attention (Editorial, Lancet Psychiatry, 2020). With the pandemic rapidly rising at the global level and so its impact on the mental health at the global scale, a pilot study was undertaken to assess the mental health status of general population post COVID-19 in India, which is a developing country with treatment gap and health care inequities.

\section{Methods}

An online survey was conducted in the month of July, 2020 in India to know how COVID-19 has affected mental health status of people post COVID-19 pandemic. The Google doc created online survey link was posted on social media like Facebook, WhatsApp and also emails were sent personally to more than 500 individuals in the category of educated professionals such as scientists, researchers, teaching, students, 
doctors etc. The responses collected through Google doc were analyzed using DASS Scale 21 method (Table 1).

\section{Results}

Of the total 27 respondents 26 were from India while one from UK. The results clearly showed that anxiety and stress were prevalent from mild to moderate level and depression in mild level, however, \% of respondents affected were low (Table 1). We received only 27 responses (a low response) in the month of July, 2020. The response of the online survey was low. There may be several reasons for this. First-people have a stigma towards mental illness, second, lack of awareness to mental health, thirdly-people think mental illness is not a serious problem since it is not life threatening, lastly, they do not want spend time in answering the form as it may not be worth to answer the survey form on mental health status. There may be several other reasons too. These reasons are very obvious if we think from Indian perspectives on mental health. Stigmatization of severe mental illness in India is common (Jadhav et. al., 2007). We found that even the category of people with good education and economic status have become vulnerable to anxiety, stress and depression post COVID-19 who do have any history of mental illness.

Discussion India for the first time launched its first National Mental Health Policy in 2014 and later revised as Mental Healthcare Act in 2017. Mental health is an integral part of health. Not only individual attributes but also socioeconomic factors and environmental factors such as national policies, social protection, etc. play a role in mental health of a person. Although poverty and low education levels are the key amongst these factors, but findings in this study indicate that the normal individuals are vulnerable to stress and anxiety due to COVID-19 pandemic. Prevention of mental disorders, and treatment and rehabilitation of people affected by mental disorders needs interventions, with focus on awareness of mental health issues. Comprehensive strategies for promotion, prevention, treatment and recovery in a whole-of-government approach to address the mental health issues is needed. Mental Health and Sustainable Development goals are now an integral part in India and its Target 3.4 is "By 2030, reduce by one third premature mortality from non-communicable diseases through prevention and treatment and promote mental health and well-being." Now to achieve such goals, India need to rather focus on the mental alarming situation due to COVID-19 situation. A recent article in Nature Medicine, Editorial, 2020 also support that surge in mental-health problems related to the COVID-19 pandemic is on the horizon and mental health crisis wave may the next one that the world need to fights. Past study has shown that one in seven Indians were affected by mental disorders in 2017 and its contribution to the total disease burden in India has almost doubled since 1990 (Dandona, 2020). Other studies also indicate the COVID19 effect on mental health and need to keep mental health in mind (Yao et. al., 2020; Lai et. al., 2020; Luo et.al. 2020; Gautam et. al., 2020; Pereira-Sanchez et.al. 2020). Besides, the mental health patients and healthcare workers, the mental well-being of the general population too are being affected post COVID-19 pandemic and is a matter of concern. An urgent attention towards a sustainable and affordable mental healthcare is needed before a second wave of mental distress hits India post COVID-19 pandemic 


\section{Conclusions}

A systematic and regular surveys need to be conducted to allow for monitoring of the mental health impact of COVID-19 in India. As the uncertain future due to COVID-19 is becoming dominant, it is high time to prepare ourselves so as to prevent the second wave of mental health crises post COVID-19 pandemic.

\section{Declarations}

\section{Acknowledgements}

The authors are thankful to Director, CSIR-NISTADS, New Delhi, India for providing support and encouragement in completing this manuscript.

\section{Ethical approval and consent to participate}

Prior consent of the participant was taken to participate in the online survey.

\section{Funding}

None

\section{Conflict of Interest}

None

\section{References}

1. Aguiar Melo MC, de Sousa Soares D. Impact of social distancing on mental health during the COVID19 pandemic: An urgent discussion. International Journal of Social Psychiatry 66(6):002076402092704.

2. Editorial. Mental health and COVID-19: change the conversation. The Lancet Psychiatry. 2020;7:463.

3. Jadhav S, Littlewood R, Ryder AG, Chakraborty A, Jain S, Barua M. Stigmatization of severe mental illness in India: Against the simple industrialization hypothesis. Indian Journal of Psychiatry. 2007;49(3):189-94.

4. Dandona L. The burden of mental disorders across the states of India: the Global Burden of Disease Study 1990-2017. The Lancet Psychiatry. 2020;7:148-61. 
5. Yao H, Chen J-H, Xu Y-F. Patients with mental health disorders in the COVID-19 epidemic. Lancet Psychiatry. 2020;7:e21.

6. Editorial. Keep mental health in mind. Nat Med, 4 May. 2020. https://doi.org/10.1038/s41591-0200914-4.

7. Lai J, Ma S, Wang Y, et al. Factors associated with mental health outcomes among health care workers exposed to coronavirus disease 2019. JAMA Netw Open. 2020;3:e203976.

8. Luoa M, Guob L, Yuc M, Jiangd W, Wange H. The psychological and mental impact of coronavirus disease 2019 (COVID19) on medical staff and general public-A systematic review and meta-analysis. Psychiatry Research. 2020;291:113190.

9. Gautam M, Thakrar A, Akinyemi E, Mahr G. Current and Future Challenges in the Delivery of Mental Healthcare during COVID-19. 2020. SN Comprehensive Clinical Medicine volume. 2020;2:865-70.

10. Pereira-Sanchez V, Adiukwu F, El Hayek S, Bytyçi DG, Gonzalez-Diaz JM, Kundadak GK, Larnaout A, Nofal M, Orsolini L, Ramalho R, Ransing R, Shalbafan M, Soler-Vidal J, Zulvia Syarif Z, Teixeira ALST, da Costa MP. COVID-19 effect on mental health: patients and workforce. The Lancet Psychiatry Vol. 2020;7:.e29e30.

\section{Table}

Table 1. Online survey using DASS Scale 21 method ( $n=27$, survey done in July, 2020, India).

(A) Demographic details of the respondents are given below:

Total number of respondents and $\quad$ Total=27, India $=26, \mathrm{UK}=1$
location

Professional category of the respondents

student (9), researcher/scientist/academician/Govt. servant/developer (10), engineer (1), teaching (6), consultant neuropsychiatric-UK (1)

Number of respondents tested positive None for COVID-19

Number of respondents suffering from

None mental illness or brain disorders before COVID-19 pandemic

Number of respondents in quarantine earlier due to CVOID-19

(B) Results of the online using DASS Scale 21 method: Depression, anxiety and stress in \% respondents $(n=27)$ are given below in number $(\%)$ : 


\begin{tabular}{|llllll|}
\hline & Normal & Mild & Moderate & Severe & Extremely Severe \\
\hline Anxiety & $23(85.19 \%)$ & $1(3.70 \%)$ & $3(11.11 \%)$ & 0 & 0 \\
\hline Stress & $17(62.96 \%)$ & $9(33.33 \%)$ & $1(3.70 \%)$ & 0 & 0 \\
\hline Depression & $22(81.48 \%)$ & $5(18.52 \%)$ & 0 & 0 & 0 \\
\hline
\end{tabular}

